\title{
Parental work characteristics and diet quality among pre-school children in dual-parent households: results from a population-based cohort in Taiwan
}

\author{
Jennifer Chun-Li Wu* \\ Early Childhood and Family Education, National Taipei University of Education, No. 134 Section 2 Heping East \\ Road, Taipei 10651, Taiwan, Republic of China
}

Submitted 10 October 2016: Final revision received 19 July 2017: Accepted 2 November 2017: First published online 13 December 2017

\begin{abstract}
Objective: To examine the relationship between parental work characteristics and diet quality among pre-school children in dual-parent households.

Design: Cross-sectional study. Parental work characteristics were measured by the types of combined parental work schedules and work hours. The main outcome variables included meal eating habits as well as 'health-conscious food' and 'unhealthy non-core food' dietary patterns derived by using principal component analysis. Sociodemographic covariates were considered to reduce confounding and selection biases.

Setting: The Taiwan Birth Cohort Study, Taiwan.

Subjects: A population-based sample of 18046 children.

Results: Multiple regression analyses indicated that compared with having both parents working standard schedules, having at least one parent who worked non-standard schedules was significantly associated with a lower likelihood of a child eating breakfast every day and a higher consumption of unhealthy non-core foods. If only one parent was employed and worked standard schedules, the children demonstrated greater odds of having home-prepared dinner most of the time. The mother's working long hours was associated with lower odds of eating breakfast every day, more frequent consumption of unhealthy non-core foods and a lower frequency of healthy food consumption.

Conclusions: The findings raise concern that parents' non-standard work schedules and mother's long working hours have negative effects on diet quality of pre-school children. Policy implications include the need for a multifaceted approach to supporting working parents so as to create healthier food environments.
\end{abstract}

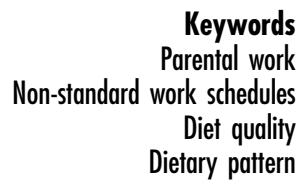

Diet quality is important for healthy growth and development of young children. Abundant evidence also indicates that poor diet quality, as assessed by food intake or eating habits, can lead to health problems such as obesity and decreased immune system function in childhood and potentially into adulthood ${ }^{(1)}$. Thus, considerable attention in epidemiological and nutritional research has been given to understanding the determinants of diet quality in children.

The family is the most influential context for early childhood development and learning, as reflected by the preponderance of discussion about diet and eating behaviours in young children being focused on family and parental factors ${ }^{(2)}$. Of these factors, behavioural and lifestyle determinants such as parental eating behaviours and modelling, home availability and accessibility of foods, child-feeding practices and parenting style have been well documented ${ }^{(3-8)}$. Social structural determinants of children's diet quality such as parental education, occupation and family income, with their linkage to parents' health knowledge, attention to health issues and economic resources in food choices, are deemed equally significant ${ }^{(2,9,10)}$. From an ecological perspective, health behaviours are embedded in and shaped by multilevel systems from the family and community to the wider socio-political system ${ }^{(11)}$. However, the role of parental employment in children's diets as part of the family system has not been addressed extensively and appears biased towards child diet-related health outcomes such as overweight and obesity ${ }^{(12-15)}$. In the few studies that explicitly examined the linkage between parents' employment status and diet quality of their children, the 
results were mixed. Although some found that less healthy eating habits or dietary quality occurred in children with full-time employed mothers ${ }^{(16-18)}$, others reported the contrary $^{(19)}$ or no significant relationship ${ }^{(20,21)}$. As for parental work characteristics, standard work schedules of both parents, greater perceived work control for fathers ${ }^{(22)}$ and lower levels of work-life stress for both parents ${ }^{(17)}$ showed positive associations with a number of healthy eating indicators in families with school-aged children and adolescents.

In many developed societies, dual-earner families are increasingly prevalent ${ }^{(23)}$ and work characteristics have become more diversified amid complex economic dynamics, such as a rise in non-standard work schedules $^{(24)}$. Therefore, empirical inquiries into parental work and children's diet quality will be of growing importance, particularly for the pre-school years, where scant literature is available. Therefore, the present study was designed to examine the relationship between parental work characteristics (work schedules and work hours) and diet quality (meal eating habits and dietary patterns) in 5-yearold Taiwanese children. The findings will also add to the existing evidence predominantly from North American, European and Australian studies.

\section{Methods}

\section{Data source and subjects}

Data for the present study came from the Taiwan Birth Cohort Study, a prospective population-based survey of children born in 2005. The Taiwan Birth Cohort Study was initiated to elucidate the health profiles of children in Taiwan and to examine early predictors of developmental health along the life course ${ }^{(25)}$. The study cohort began with 24200 live births drawn from the national birth report database using two-stage stratified random sampling, and $21248(87.80 \%)$ infants participated in the first survey at 6 months old. Before children reached school age, follow-up surveys were conducted at 18 and 36 months and at 5 years of age. Because the present study focused on diet quality of pre-school children living in dual-parent households, the sample consisted of 18046 subjects who had completed the 5-year survey and whose parents were married at the time of the interview. The survey questionnaires were designed to collect age-relevant information about each child's physical growth, medical history and behavioural development. A range of social and physical exposures that young children may experience in their family, childcare and community environments were also queried ${ }^{(25)}$. To obtain accurate and reliable information, the mothers or primary caregivers were designated as the respondents for faceto-face interviews. For the 5-year survey interviews, 98.55\% were conducted with mothers, $0.90 \%$ with fathers and $0.43 \%$ with grandparents.

\section{Measures}

Parental work schedules

Parental work characteristics captured during the study interview included the times and hours worked at current jobs. A question was first asked about the employment status of the mother and those employed further indicated the times they usually worked with four response categories: day shift, night shift, day-night rotating shift, and both days and nights. The mother's work schedule was coded as 'standard' if she worked day shifts and as 'nonstandard' otherwise. Mothers who reported not working were coded as 'not employed'. Parallel inquiries and data were obtained regarding the father's work schedule. Because parental employment and work schedule decisions are generally not independent, a variable was created to reflect the combination of parents' work schedules as one of the primary predictors in the study. Based on the above information, six categories of combined parental work schedules were defined: (i) both working standard; (ii) one working standard and one non-standard; (iii) both working non-standard; (iv) only one employed and working standard; (v) only one employed and working non-standard; and (vi) neither parent employed. If there was no information on one of the parents' work schedule, a missing value was coded for this variable.

\section{Parental work hours}

Parents' work hours affect family life and it is high total work hours that raises concern for the well-being of children ${ }^{(18,26)}$. Therefore, the average weekly work hours of each parent were recoded into four categories: $\leq 20$, 21-40, 41-60 and >60 h. The cut-off points were chosen with reference to the statutory working hours ( $40 \mathrm{~h} /$ week) stipulated in Taiwan's Labour Standards Act and the average hours of work for part-time employees (19.2 h/ week) based on the 2014 Manpower Utilization Survey ${ }^{(27)}$.

\section{Diet quality}

The dietary quality of pre-school children was conceptualized in terms of meal eating habits and dietary patterns. Meal eating habits were measured by the frequency of eating breakfast and having home-prepared dinner. Preparation of food at home has been suggested to predict healthier diets ${ }^{(28,29)}$. Dichotomous outcome variables (yes/no) were created to record whether the child was reported to 'eat breakfast every day' and 'have home-prepared dinner most of the time'.

Foods are consumed in combination and there is usually a high correlation between consumption of certain nutrients or foods. Traditional focus on single nutrients or food groups in nutritional epidemiology therefore ignores the complex nature of the human diet and fails to depict the overall diet that is more useful in examining its linkage with a variety of health outcomes ${ }^{(30)}$. Owing to this concern, dietary pattern analysis - with which combinations of 
multiple food components by consumption frequency and/or amount are identified to capture the characteristics and habits of diet - has therefore been proposed ${ }^{(30,31)}$. Two major approaches can be used to derive dietary patterns. A variable-based approach involves grouping food variables into a reduced dimensionality reflecting major dietary traits, while an individual-based approach places study subjects into distinct groups with regard to shared characteristics of food consumption $^{(32)}$. The present study was based on a variable approach using principal component analysis because it allows the comparison of individual variation in food consumption level and has been the most widely applied method in dietary pattern analysis ${ }^{(30,33-35)}$. To assess children's diet, the respondents were asked to rate 'How often does your child eat the following foods?', with eleven food groups presented: (i) meats; (ii) seafood such as fish and shrimp; (iii) beans/bean products; (iv) eggs; (v) grains/starchy roots; (vi) vegetables; (vii) fruits; (viii) dairy products; (ix) burgers/pizza/fried chicken; (x) candy/cookies/cakes; and (xi) beverages/Coca-Cola/ soft drinks (tea, coffee and mineral water not included). The first eight items covered basic food groups defined in the Daily Dietary Guidelines for Taiwanese ${ }^{(36)}$, while the other three items were chosen as they are common processed and snack foods high in fat or sugar. Ratings were on a five-point frequency scale including 'never', 'less than once per week', ' 1 to 2 times per week', ' 3 to 5 times per week' and 'every day'. Using the five ordinal levels of food group consumption, principal component analysis with varimax was conducted to generate the dietary patterns. The Kaiser-Meyer-Olkin value was 0.70 and the Bartlett's test for sphericity was highly significant. Four components with an eigenvalue $\geq 1$ were extracted, accounting for $53.15 \%$ of total variance. Two dietary patterns were of special attention (Table 1): one represented an 'unhealthy non-core food' pattern (eigenvalue $=1.56$ ) characterized by high intakes of burgers/pizza/fried chicken, candy/ cookies/cakes and beverages/Coca-Cola/soft drinks; while the other was characterized as a 'health-conscious food' pattern (eigenvalue $=1.42$ ) with high intakes of vegetables, fruits, grains/starchy roots. Regression-based component scores as a linear combination of food group consumption were computed for each component to represent dietary pattern scores. A greater dietary pattern score indicated that the child more frequently consumed the foods of that specific dietary pattern.

Table 1 Descriptive statistics for eating behaviours and food group consumption, and dietary patterns derived by principal component analysis with factor loadings, in 5-year-old pre-school children $(n$ 18046) in dual-parent households, Taiwan Birth Cohort Study

\begin{tabular}{|c|c|c|c|c|}
\hline Meal eating habits & $n$ & $\%$ & & \\
\hline \multicolumn{5}{|l|}{ Frequency of eating breakfast (times/week) } \\
\hline Never or $<1$ & 93 & 0.52 & & \\
\hline 1 to 2 & 216 & 1.20 & & \\
\hline 3 to 5 & 362 & $2 \cdot 00$ & & \\
\hline Almost every day & 17375 & $96 \cdot 28$ & & \\
\hline \multicolumn{5}{|l|}{ Frequency of having home-prepared dinner } \\
\hline Prepared at home most of the time & 13047 & $72 \cdot 30$ & & \\
\hline Half of the time & 3100 & $17 \cdot 18$ & & \\
\hline Eating out or takeout meals most of the time & 1895 & 10.50 & & \\
\hline Missing & 4 & 0.02 & & \\
\hline & & & \multicolumn{2}{|c|}{ Dietary pattern } \\
\hline Frequency of consumption by food group & $n$ & $\%$ & 'Unhealthy non-core food' & 'Health-conscious food' \\
\hline \multicolumn{5}{|l|}{ Core foods (never or $<1$ time/week) } \\
\hline Grains/starchy roots & 32 & 0.18 & & 0.63 \\
\hline Meats & 530 & 2.94 & & \\
\hline Seafood (e.g. fish, shrimp) & 1161 & 6.44 & & \\
\hline Beans/bean products & 1495 & 8.28 & & \\
\hline Eggs & 527 & $2 \cdot 92$ & & \\
\hline Vegetables & 230 & 1.28 & & 0.73 \\
\hline Fruits & 304 & 1.69 & & 0.59 \\
\hline Dairy products & 994 & 5.50 & & \\
\hline \multicolumn{5}{|l|}{ Non-core foods (almost every day) } \\
\hline Burgers/pizza/fried chickens & 85 & 0.47 & 0.64 & \\
\hline Candy/cookies/cake & 4882 & $27 \cdot 06$ & 0.71 & \\
\hline Beverages/Coca-Cola/soft drinks & 1404 & $7 \cdot 78$ & 0.76 & \\
\hline \multirow[t]{2}{*}{ Variance explained (\%) } & \multirow{2}{*}{\multicolumn{2}{|c|}{ Dietary pattern adherence score }} & $19 \cdot 61$ & 9.61 \\
\hline & & & & \\
\hline \multicolumn{5}{|l|}{ ‘Unhealthy non-core food’ } \\
\hline Mean & \multirow{2}{*}{\multicolumn{2}{|c|}{$\begin{array}{r}-4.70 \\
3.81\end{array}$}} & & \\
\hline SD & & & & \\
\hline \multicolumn{5}{|l|}{ 'Health-conscious food' } \\
\hline Mean & \multirow{2}{*}{\multicolumn{2}{|c|}{-13.58}} & & \\
\hline SD & 1.98 & & & \\
\hline
\end{tabular}


Sociodemographic covariates

A set of child and family sociodemographic characteristics previously found to be associated with children's dietary patterns ${ }^{(2,7-8,10)}$ were controlled for in the analytical models. These variables included the child's sex, paternal and maternal education (formal schooling $\leq 9$, 10-12 and $\geq 13$ years), family monthly income ( $<30000$, 30000 69999 and $\geq 70000$ New Taiwan Dollars), pre-school attendance and family informal care resources indicated by the co-residence of any adult(s) in addition to parents in the household.

\section{Statistical analyses}

Multiple regression analysis was performed to examine the relationship between parental work characteristics and children's diet quality using the statistical software package SAS version 9.4. Regression was performed for parental combined work schedules and long work hours on the outcome variables (Model 1) and further adjusted for covariates (Model 2) to reduce potential confounding and selection biases. Logistic regression was applied to estimate the OR and 95\% CI for optimal eating habits (eating breakfast every day and having home-prepared dinner most of the time), whereas multiple linear regression models were fitted for predicting children's dietary patterns ('unhealthy non-core food' and 'health-conscious food' dietary pattern scores).

\section{Results}

\section{Subjects' characteristics}

Table 2 presents the sociodemographic characteristics of subject children and their parents. Of the subjects, $52.44 \%$ were boys, the majority $(97 \cdot 21 \%)$ went to childcare and $12.42 \%$ had at least one adult other than parents residing in the household. Almost half of the mothers (47.57\%) and fathers $(48.26 \%)$ had completed some college education. Of the mothers, $13.05 \%$ were foreign-born ( $4.51 \%$ from China and $8.54 \%$ from South-East Asian or other countries) and were mainly marriage migrants.

\section{Parental work characteristics}

As shown in Table 2, about a quarter (25.91\%) of mothers and only $3.82 \%$ of fathers in dual-parent households were unemployed. Parents working non-standard schedules was not uncommon (20.74\% for mothers and $37.30 \%$ for fathers) and the rates of working over $40 \mathrm{~h}$ weekly were $56.71 \%$ for employed mothers and $69.22 \%$ for employed fathers. The data combining parental work schedules indicated that having both parents working standard schedules was most prevalent (35.19\%), followed by one working standard and one non-standard (24.62\%), and finally both parents working non-standard schedules (10.88\%).
Table 2 Characteristics of the study population of 5-year-old preschool children ( $n$ 18046) and their parents in dual-parent households, Taiwan Birth Cohort Study

\begin{tabular}{|c|c|c|}
\hline & $n$ & $\%$ \\
\hline Sex, boys & 9463 & 52.44 \\
\hline Pre-school attendance & 17542 & $97 \cdot 21$ \\
\hline Co-residence of adult(s) other than parents & 2241 & $12 \cdot 42$ \\
\hline \multicolumn{3}{|l|}{ Maternal education } \\
\hline$\leq 9$ years & 2436 & 13.50 \\
\hline $10-12$ years & 7000 & 38.79 \\
\hline$\geq 13$ years & 8584 & 47.57 \\
\hline Missing & 26 & 0.14 \\
\hline \multicolumn{3}{|l|}{ Paternal education } \\
\hline$\leq 9$ years & 2259 & $12 \cdot 52$ \\
\hline $10-12$ years & 7032 & 38.97 \\
\hline$\geq 13$ years & 8709 & $48 \cdot 26$ \\
\hline Missing & 46 & 0.25 \\
\hline \multicolumn{3}{|l|}{ Family monthly income } \\
\hline$<30000$ NTD & 1849 & $10 \cdot 25$ \\
\hline 30 000-69999 NTD & 9222 & $51 \cdot 10$ \\
\hline$\geq 70000$ NTD & 6873 & 38.09 \\
\hline Missing & 102 & 0.57 \\
\hline \multicolumn{3}{|l|}{ Mother's place of origin } \\
\hline Taiwan & 15691 & 86.95 \\
\hline China & 813 & 4.51 \\
\hline Others & 1542 & 8.54 \\
\hline \multicolumn{3}{|l|}{ Mother's work schedule } \\
\hline Standard work schedule & 9543 & $52 \cdot 88$ \\
\hline Non-standard work schedule & 3742 & $20 \cdot 74$ \\
\hline Not employed & 4676 & 25.91 \\
\hline Missing & 85 & 0.47 \\
\hline Mother's weekly work hours & $(n$ 13285) & \\
\hline$\leq 20 \mathrm{~h}$ & 558 & $4 \cdot 20$ \\
\hline $21-40 \mathrm{~h}$ & 5183 & 39.01 \\
\hline $41-60 \mathrm{~h}$ & 6282 & 47.29 \\
\hline$>60 \mathrm{~h}$ & 1252 & 9.42 \\
\hline Missing & 0 & 0.08 \\
\hline \multicolumn{3}{|l|}{ Father's work schedule } \\
\hline Standard work schedule & 10578 & 58.62 \\
\hline Non-standard work schedule & 6732 & $37 \cdot 30$ \\
\hline Not employed & 690 & 3.82 \\
\hline Missing & 46 & 0.25 \\
\hline Father's weekly work hours & $(n 17310)$ & \\
\hline$\leq 20 \mathrm{~h}$ & 214 & 1.23 \\
\hline$\overline{21-40 h}$ & 5073 & $29 \cdot 31$ \\
\hline $41-60 \mathrm{~h}$ & 9019 & $52 \cdot 10$ \\
\hline$>60 \mathrm{~h}$ & 2963 & $17 \cdot 12$ \\
\hline Missing & 41 & 0.24 \\
\hline \multicolumn{3}{|l|}{ Parental combined work schedules } \\
\hline Both working standard & 6351 & $35 \cdot 19$ \\
\hline One working standard, one non-standard & 4443 & 24.62 \\
\hline Both working non-standard & 1964 & $10 \cdot 88$ \\
\hline Only one employed, working standard & 2923 & $16 \cdot 20$ \\
\hline Only one employed, working non-standard & 2061 & 11.42 \\
\hline Neither parent employed & 182 & 1.01 \\
\hline Missing & 122 & 0.68 \\
\hline
\end{tabular}

NTD, New Taiwan Dollars.

\section{Children's diet quality}

Table 1 shows the descriptive statistics for meal eating habits and food group consumption among 5-year-old children in the present study. Most children (96.28\%) ate breakfast almost every day and $72 \cdot 30 \%$ had homeprepared dinner most of the time. For the consumption of core foods, the proportions of children reported with a frequency of 'never' or 'less than once per week' were generally low and highest for beans/bean products 
( $8.28 \%)$, seafood $(6.44 \%)$ and dairy products $(5.50 \%)$. With respect to non-core foods, a rather small group $(0.47 \%)$ of children had burgers/pizza/fried chicken almost every day, while over a quarter (27.06\%) reported having candy/cookies/cake daily. The dietary pattern scores were in the range of -13.58 to 1.98 for the "healthconscious food' pattern and -4.70 to $3 \cdot 81$ for the 'unhealthy non-core food' pattern.

\section{Logistic regression analyses: meal eating behaviours}

Table 3 presents the results from logistic regression analyses of parental work schedules and work hours on children's breakfast habits. Model 1 shows that children with at least one parent working non-standard schedules were significantly less likely to eat breakfast every day than those whose parents both worked standard schedules. Father's long weekly working hours at $41-60 \mathrm{~h}$, as compared with $\leq 20 \mathrm{~h}$, was associated with an increased likelihood of the child having breakfast every day. After adjustment for sociodemographic covariates in Model 2, the associations between parental work schedules and children's breakfast habits remained. The OR were 0.75 (95\% CI 0.60, 0.93) for children with one parent working standard and the other non-standard, 0.72 (95\% CI 0.54 , $0.96)$ with both parents working non-standard and 0.62 (95\% CI $0.42,0.92$ ) for those with only one parent employed and working non-standard schedules. The work hours of the father were no longer associated with the child's breakfast eating habits, while mother's long work hours (41-60 h/week) became significantly predictive of the child not having breakfast every day (OR $=0.69,95 \%$ CI $0.48,0.98$ ).

As shown in Table 3 (Model 1), the likelihood of children having home-prepared dinner most of the time was greater for those with only one parent employed and working standard schedules than for those whose parents both worked standard schedules. In the adjusted Model 2, the above association remained statistically significant $(\mathrm{OR}=1.31,95 \% 1.10,1.56)$. Interestingly, longer work hours of fathers were significantly associated with increased odds of children having home-prepared dinner

Table 3 Logistic regression analysis of parental work schedules and meal eating habits; unadjusted and adjusted OR and $95 \% \mathrm{Cl}$ for eating breakfast every day and having home-prepared dinner most of the time among 5-year-old pre-school children ( $n$ 18046) in dual-parent households, Taiwan Birth Cohort Study

\begin{tabular}{|c|c|c|c|c|c|c|c|c|}
\hline & \multicolumn{4}{|c|}{ Eating breakfast every day } & \multicolumn{4}{|c|}{ Having home-prepared dinner most of the time } \\
\hline & \multicolumn{2}{|c|}{ Model 1} & \multicolumn{2}{|c|}{ Model 2} & \multicolumn{2}{|c|}{ Model 1} & \multicolumn{2}{|c|}{ Model 2} \\
\hline & OR & $95 \% \mathrm{Cl}$ & OR & $95 \% \mathrm{Cl}$ & OR & $95 \% \mathrm{Cl}$ & OR & $95 \% \mathrm{Cl}$ \\
\hline \multicolumn{9}{|c|}{ Parental work schedules (Ref.: Both working standard) } \\
\hline One working standard, one non-standard & 0.75 & $0.61,0.93$ & 0.75 & $0.60,0.93$ & 0.94 & $0.86,1.02$ & 0.92 & $0.85,1.01$ \\
\hline Both working non-standard & 0.70 & $0.52,0.93$ & 0.72 & $0.54,0.96$ & 0.91 & $0.81,1.03$ & 0.90 & $0.79,1.02$ \\
\hline Only one employed, working standard & 0.77 & $0.52,1.14$ & 0.82 & $0.56,1.20$ & 1.36 & $1.15,1.62$ & 1.31 & $1.10,1.56$ \\
\hline Only one employed, working non-standard & 0.63 & $0.43,0.95$ & 0.62 & $0.42,0.92$ & 0.89 & $0.74,1.06$ & 0.88 & $0.74,1.06$ \\
\hline Neither parent employed & 0.64 & $0.28,1.50$ & 0.70 & $0.30,1.64$ & 1.31 & $0.84,2.04$ & 1.26 & $0.80,1.97$ \\
\hline \multicolumn{9}{|l|}{ Mother's weekly work hours (Ref.: $\leq 20 \mathrm{~h}$ ) } \\
\hline $21-40 \mathrm{~h}$ & $1 \cdot 10$ & $0.76,1.60$ & 0.84 & $0.58,1.22$ & 0.94 & $0.80,1.10$ & 1.04 & $0.89,1.23$ \\
\hline $41-60 \mathrm{~h}$ & 0.84 & $0.59,1.20$ & 0.69 & $0.48,0.98$ & 0.98 & $0.84,1.15$ & 1.02 & $0.87,1.19$ \\
\hline$>60 \mathrm{~h}$ & 0.97 & $0.63,1.50$ & 0.94 & $0.61,1.46$ & 0.96 & $0.79,1.16$ & 0.88 & $0.72,1.07$ \\
\hline \multicolumn{9}{|l|}{ Father's weekly work hours (Ref.: $\leq 20 \mathrm{~h}$ ) } \\
\hline $21-40 \mathrm{~h}$ & 1.55 & $1.04,2.29$ & 0.94 & $0.63,1.42$ & 1.02 & $0.84,1.24$ & $1 \cdot 28$ & $1.05,1.57$ \\
\hline $41-60 \mathrm{~h}$ & 1.69 & $1.16,2.47$ & 1.01 & $0.68,1.50$ & 0.99 & $0.82,1.19$ & 1.29 & $1.06,1.56$ \\
\hline$>60 \mathrm{~h}$ & 1.34 & $0.89,2.01$ & 0.85 & $0.56,1.30$ & 0.89 & $0.73,1.08$ & $1 \cdot 12$ & $0.91,1.37$ \\
\hline \multicolumn{9}{|l|}{ Child's sex (Ref.: girls) } \\
\hline Boys & & & $1 \cdot 18$ & $1.01,1.38$ & & & 1.02 & $0.95,1.08$ \\
\hline \multicolumn{9}{|l|}{ Maternal education (Ref.: $\leq 9$ years) } \\
\hline $10-12$ years & & & 1.24 & $0.97,1.60$ & & & 0.91 & $0.80,1.04$ \\
\hline$\geq 13$ years & & & 1.45 & $1.07,1.96$ & & & 0.92 & $0.79,1.06$ \\
\hline \multicolumn{9}{|l|}{ Paternal education (Ref.: $\leq 9$ years) } \\
\hline $10-12$ years & & & 1.26 & $1.00,1.57$ & & & 1.05 & $0.94,1.18$ \\
\hline$\geq 13$ years & & & 1.47 & $1.12,1.94$ & & & 1.04 & $0.91,1.19$ \\
\hline \multicolumn{9}{|l|}{ Family monthly income (Ref.: $<30000$ NTD) } \\
\hline $30000-69999$ NTD & & & 1.85 & $1.47,2.33$ & & & 0.85 & $0.74,0.97$ \\
\hline$\geq 70000$ NTD & & & $2 \cdot 22$ & $1.67,2.97$ & & & 0.66 & $0.57,0.77$ \\
\hline \multicolumn{9}{|l|}{ Mother's nationality of origin (Ref.: Taiwan) } \\
\hline China & & & 1.40 & $0.95,2.06$ & & & 1.96 & $1.60,2.41$ \\
\hline Others & & & 1.36 & $1.01,1.83$ & & & 1.54 & $1.32,1.80$ \\
\hline Pre-school attendance & & & 3.19 & $2.37,4.29$ & & & 0.92 & $0.74,1.13$ \\
\hline Co-residence of adult(s) other than parents & & & 0.84 & $0.68,1.05$ & & & 1.63 & $1.46,1.83$ \\
\hline Likelihood $\chi^{2}$ & & $46 \cdot 04$ & & $192 \cdot 76$ & & 94.66 & & $396 \cdot 24$ \\
\hline$P$ value & & $<0.001$ & & $<0.001$ & & $<0.001$ & & $<0.001$ \\
\hline
\end{tabular}

Ref., reference group; NTD, New Taiwan Dollars. 
(21-40 h: OR $=1 \cdot 28,95 \%$ CI $1 \cdot 05,1 \cdot 57 ; 41-60 \mathrm{~h}:$ OR $=1 \cdot 29$, $95 \%$ CI 1.06, 1.56), while mother's work hours were not found to be a significant predictor.

\section{Linear regression analyses: dietary patterns}

Table 4 shows the results of multiple linear regression analyses examining the association between parental work schedules and the children's dietary patterns. As indicated by both unadjusted models (Model 1), parental work schedules and work hours could predict children's consumption of unhealthy non-core food and health-conscious food. After children's sex and family sociodemographic characteristics were controlled for (Model 2), having at least one parent working non-standard schedules was significantly associated with higher 'unhealthy non-core food' pattern scores compared with having parents both working standard schedules. The regression coefficients were $0 \cdot 13(P<0 \cdot 001)$ for children with both parents working non-standard schedules and 0.08 with one parent working standard and the other nonstandard schedules $(P<0.001)$ as well as with only one employed and working non-standard schedules $(P=0 \cdot 05)$. However, parental work schedules became non-significantly associated with 'health-conscious food' pattern scores. In addition, compared with children whose mothers worked no more than $20 \mathrm{~h}$ weekly, those with mothers who had longer weekly work hours scored significantly higher on the 'unhealthy non-core food' dietary pattern $(21-40 \mathrm{~h}$ : $\beta=0 \cdot 09$, $P=0.01 ; \quad 41-60 \mathrm{~h}: \quad \beta=0.17, \quad P<0.001 ; \quad>60 \mathrm{~h}: \quad \beta=0.15$, $P<0 \cdot 001)$, but lower on the 'health-conscious food' dietary pattern $(\beta=-0 \cdot 10$ to $-0 \cdot 12, P<0 \cdot 01)$.

\section{Discussion}

\section{Main findings}

The present study demonstrated significant associations between parental work schedules and diet quality among pre-school children after adjustment for sociodemographic covariates in a population sample of 5 -year-olds in Taiwan.

When either or both parents worked non-standard schedules, their pre-school children tended to consume

Table 4 Regression analysis of parental work schedules and dietary patterns; unstandardized $\beta$ coefficients and $P$ values for 'unhealthy non-core food' and 'health-conscious food' dietary scores among 5-year-old pre-school children ( $n$ 18046) in dual-parent households, Taiwan Birth Cohort Study

\begin{tabular}{|c|c|c|c|c|c|c|c|c|}
\hline & \multicolumn{4}{|c|}{ 'Unhealthy non-core food' } & \multicolumn{4}{|c|}{ 'Health-conscious food' } \\
\hline & \multicolumn{2}{|c|}{ Model 1} & \multicolumn{2}{|c|}{ Model 2} & \multicolumn{2}{|c|}{ Model 1} & \multicolumn{2}{|c|}{ Model 2} \\
\hline & $\beta$ & $P$ value & $\beta$ & $P$ value & $\beta$ & $P$ value & $\beta$ & $P$ value \\
\hline \multicolumn{9}{|c|}{ Parental work schedules (Ref.: Both working standard) } \\
\hline One working standard, one non-standard & 0.08 & $<0.001$ & 0.08 & $<0.001$ & -0.03 & 0.13 & -0.03 & $0 \cdot 17$ \\
\hline Both working non-standard & $0 \cdot 13$ & $<0.001$ & $0 \cdot 13$ & $<0.001$ & -0.03 & 0.34 & -0.02 & 0.39 \\
\hline Only one employed, working standard & 0.02 & 0.60 & -0.02 & 0.58 & -0.02 & 0.54 & 0.01 & 0.87 \\
\hline Only one employed, working non-standard & 0.09 & 0.03 & 0.08 & 0.05 & -0.05 & 0.21 & -0.04 & 0.32 \\
\hline Neither parent employed & -0.08 & 0.40 & -0.09 & 0.30 & -0.20 & 0.04 & -0.18 & 0.06 \\
\hline \multicolumn{9}{|l|}{ Mother's weekly work hours (Ref.: $\leq 20 \mathrm{~h}$ ) } \\
\hline $21-40 \mathrm{~h}$ & -0.00 & 0.95 & 0.09 & 0.01 & -0.05 & $0 \cdot 17$ & -0.11 & $<0.01$ \\
\hline $41-60 \mathrm{~h}$ & $0 \cdot 14$ & $<0.001$ & 0.17 & $<0.001$ & -0.07 & 0.04 & $-0 \cdot 10$ & $<0.01$ \\
\hline$>60 \mathrm{~h}$ & 0.25 & $<0.001$ & $0 \cdot 15$ & $<0.001$ & $-0 \cdot 16$ & $<0.001$ & -0.12 & $<0.01$ \\
\hline \multicolumn{9}{|l|}{ Father's weekly work hours (Ref.: $\leq 20 \mathrm{~h}$ ) } \\
\hline $21-40 \mathrm{~h}$ & -0.20 & $<0.001$ & -0.02 & 0.58 & 0.06 & 0.17 & -0.05 & 0.23 \\
\hline $41-60 \mathrm{~h}$ & -0.21 & $<0.001$ & -0.03 & 0.46 & 0.04 & 0.32 & -0.07 & 0.11 \\
\hline$>60 \mathrm{~h}$ & $-0 \cdot 16$ & $<0.001$ & -0.03 & 0.57 & -0.02 & 0.62 & -0.11 & 0.02 \\
\hline \multicolumn{9}{|l|}{ Child's sex (Ref.: girls) } \\
\hline Boys & & & 0.04 & $<0.01$ & & & -0.07 & $<0.001$ \\
\hline \multicolumn{9}{|l|}{ Maternal education (Ref.: $\leq 9$ years) } \\
\hline $10-12$ years & & & -0.11 & $<0.001$ & & & 0.09 & $<0.001$ \\
\hline$\geq 13$ years & & & -0.29 & $<0.001$ & & & 0.17 & $<0.001$ \\
\hline \multicolumn{9}{|l|}{ Paternal education (Ref.: $\leq 9$ years) } \\
\hline 10-12 years & & & -0.08 & $<0.01$ & & & 0.03 & $0 \cdot 17$ \\
\hline \multirow{2}{*}{\multicolumn{9}{|c|}{ Family monthly income (Ref.: <30000 NTD) }} \\
\hline & & & & & & & & \\
\hline $30000-69999$ NTD & & & $-0 \cdot 10$ & $<0.001$ & & & 0.08 & $<0.01$ \\
\hline$\geq 70000$ NTD & & & -0.22 & $<0.001$ & & & 0.20 & $<0.001$ \\
\hline \multicolumn{9}{|l|}{ Mother's nationality of origin (Ref.: Taiwan) } \\
\hline China & & & -0.20 & $<0.001$ & & & 0.14 & $<0.001$ \\
\hline Others & & & 0.07 & 0.02 & & & 0.02 & 0.45 \\
\hline Pre-school attendance & & & 0.08 & 0.05 & & & 0.15 & 0.001 \\
\hline Co-residence with adult(s) other than parents & & & -0.01 & 0.54 & & & 0.01 & 0.80 \\
\hline$F$ value & \multirow{3}{*}{\multicolumn{2}{|c|}{$\begin{array}{c}23.75 \\
0.014 \\
<0.001\end{array}$}} & \multirow{2}{*}{\multicolumn{2}{|c|}{$\begin{array}{c}58.92 \\
0.066 \\
<0.001\end{array}$}} & \multirow{3}{*}{\multicolumn{2}{|c|}{$\begin{array}{c}5.54 \\
0.003 \\
<0.001\end{array}$}} & \multirow{2}{*}{\multicolumn{2}{|c|}{$\begin{array}{c}18 \cdot 34 \\
0.021\end{array}$}} \\
\hline Adjusted $R^{2}$ & & & & & & & & \\
\hline$P$ value & & & \multicolumn{2}{|c|}{$<0.001$} & & & \multicolumn{2}{|c|}{$<0.001$} \\
\hline
\end{tabular}

Ref., reference group; NTD, New Taiwan Dollars. 
more unhealthy non-core foods and not to eat breakfast every day. The results echoed previous literature in relation to use of fast-food restaurants among school-aged children $^{(22)}$. Parents may work at non-standard times for various reasons, fulfilling structural demands of the labour market or serving as a strategy to meet child caregiving needs ${ }^{(37)}$. Despite the reasons, reviews of empirical studies have concluded that if the parents have nonstandard work schedules, there are negative effects on a range of behavioural and health outcomes of their children. This presumably is mediated by parents' time, mental health, work-family stress, parent-child interactions and parenting ${ }^{(24,38)}$. As examples relevant to children's diets, non-standard work arrangements pose challenges for parents to support family routines such as preparing meals at regular times and having meals together, or to monitor the child's food intake consistently. This may result in children being more likely to resort to snack foods as a meal alternative or to have fast foods for convenience. Nevertheless, in the present study, parental non-standard work schedules did not predict lower scores on the health-conscious dietary pattern in their children. This finding was in line with earlier studies suggesting differences in determinants of consumption between healthy and unhealthy non-core foods ${ }^{(5,7)}$. Nearly all children in the present study went to a pre-school where nutritionally balanced meals were provided and regulated by law ${ }^{(39)}$. This could be one of the reasons why having a parent with a non-standard work schedule did not interfere with young children having an adequate fruit and vegetable intake.

As for predictions based on parental work hours, having a mother who worked long hours was significantly associated with poorer diet quality in children, reflected by being less likely to eat breakfast, consuming more unhealthy non-core foods and less healthy foods. This can be anticipated because longer work hours of parents can indicate that less time is available for meal planning, preparation and food shopping ${ }^{(17,38)}$, especially for mothers who carry out relatively more housework duties in current Taiwanese society ${ }^{(40)}$. However, the relationship was not described by a typical dose-effect curve. People working long hours constitute a heterogeneous group with respect to their sociodemographic status, work conditions and employment type ${ }^{(41)}$. For example, compared with paid employees, the self-employed or those working in a family business are more likely to put in long hours but have greater flexibility in the timing and location of work for work-family balance ${ }^{(42,43)}$. This may explain why the highest bracket of mother's work hours (i.e. >60 h/week) may not necessarily predict poorest diet in children.

Another finding of note was that mother's long work hours did not predict children being less likely to eat home-prepared dinner. Time constraint due to labour force participation of married women has long been the dominant explanation for the decline in home meal preparation. However, more recent literature on the decision of dinner preparation at home pointed to a more complex picture in relation to such factors as the food market (e.g. availability of convenience food products, fast-food restaurants) and personal attitudes (e.g. enjoyment or self-efficacy of cooking, cost-effectiveness of preparing meal at home $v$. eating out ${ }^{(28,44,45)}$. All these may contribute to mothers' practices of home dinner preparation regardless of their work hours.

It is also worthy of attention that mother's nationality of origin was consistently related children's diet quality, with a higher likelihood of having home-prepared dinner for children of non-Taiwanese mothers the most pronounced finding. Mothers not originally from Taiwan were mainly migrants from less developed countries (e.g. China, Vietnam and Indonesia) married to socially or functionally disadvantaged men in Taiwan ${ }^{(46)}$. Because family socioeconomic covariates were controlled for in the regression models, the finding may be more appropriately attributed to cultural explanations in relation to health beliefs, values placed on family meals and representation of domestic female roles ${ }^{(47,48)}$. An in-depth discussion of this finding is beyond the scope of the present study and can be further addressed in future research.

\section{Strengths and limitations}

Strengths of the current study include the use of a large-scale representative birth cohort with high response rates in all follow-up surveys, which rendered it possible to investigate different combinations of parental work schedules and to secure the generalizability of the findings. Another strength relates to the expanded scope that captures various work characteristics of both fathers and mothers. Previous studies focused largely on maternal employment and limited aspects of the employment status (e.g. employed $v$. unemployed and full-time $v$. part-time employment).

Such a research framework should provide more useful insights given the globalized and increasingly marketdriven world economy as well as a growing emphasis on the paternal role in child rearing.

However, the present study is not without limitations and the first concerns the definition of non-standard work schedules of parents. Conceptually, non-standard work schedules refer to work hours not fitting in a typical daytime, five-day working week (Monday to Friday), which can include evening shifts, night shifts, rotating shifts, weekend work and irregular hours ${ }^{(24,38)}$. In the present study, the survey question selected to measure parental work schedules asked only about day or/and night shifts in a day, but no information about weekend work or irregular hours was sought and recorded. Thus, the prevalence of parents who worked non-standard work schedules might have been underestimated. Second, dietary assessment of children by parental reporting could elicit socially desirable responses, potentially leading to 
biased estimates of association. With the existing survey data set, unfortunately, the study was not able to include any social desirability measures to adjust for potential biases. However, participants in the longitudinal study have had follow-up interviews at least twice, and they may not associate dietary assessment with specific disease status as in case-control studies. Social desirability bias in such a case could be less of a concern ${ }^{(49)}$.

\section{Policy implications and future research}

Parental work is a key social determinant of child health, yet it has not drawn sufficient attention ${ }^{(50)}$. How parental work characteristics are linked to the quality of their children's diets, particularly in the pre-school years when eating habits are shaped, remains even less explored. The present findings highlight the negative effects of parents' non-standard work schedules and long work hours on both meal eating habits and dietary intake of children, with family sociodemographic factors held constant. Multifaceted policy efforts should be geared towards supporting both parents so as to create healthier family food environments. This can be achieved by giving parents greater control and choices over work times ${ }^{(37)}$, placing limits on work hours ${ }^{(51)}$, coordinating after-school care and community resources during parents' non-standard work hours ${ }^{(24)}$, and enhancing the education of parents about childhood nutritional and health needs. Future research is needed to disentangle the interplay between parental work characteristics and diet quality of young children as modified by contextual factors such as family income and after-school care arrangements. Further application of longitudinal panel data will also help to elucidate the causal mechanisms linking parents' work characteristics and family food environments that are critical to the diet quality and dietary behaviour of young children.

\section{Acknowledgements}

Acknowledgements: This study was based on longitudinal data from the Taiwan Birth Cohort Study, funded by the Health Promotion Administration, Ministry of Health and Welfare. The author gratefully acknowledges the time and continuing support from the participating families. Financial support: This research received no specific grant from any funding agency in the public, commercial or notfor-profit sectors. Conflict of interest: None. Authorship: The sole author, J.C.L.W. formulated the research questions, designed the study, analysed the data, interpreted the results and wrote the manuscript. Ethics of buman subject participation: This research used data of a longitudinal survey study which was conducted according to the guidelines laid down in the Declaration of Helsinki and all procedures involving human subjects were approved by the Institutional Review Board of the Directorate-General of Budget, Accounting, and Statistics, Executive Yuan
(No. 94-C3-09400005257). Written informed consent was obtained from all survey respondents.

\section{References}

1. Darnton-Hill I, Nishida C \& James WPT (2004) A life course approach to diet, nutrition and the prevention of chronic diseases. Public Health Nutr 7, 101-121.

2. Patrick H \& Nicklas TA (2005) A review of family and social determinants of children's eating patterns and diet quality. J Am Coll Nutr 24, 83-92.

3. Wyse R, Campbell E, Nathan E et al. (2011) Associations between characteristics of the home food environment and fruit and vegetable intake in preschool children: a crosssectional study. BMC Public Health 11, 938.

4. McPhie S, Skouterisa H, Fuller-Tyszkiewicz M et al. (2012) Maternal predictors of preschool child-eating behaviours, food intake and body mass index: a prospective study. Early Child Dev Care 182, 999-1014.

5. McGowan L, Croker H, Wardle J et al. (2012) Environmental and individual determiants of core and non-core food and drink intake in preschool-aged children in the United Kindom. Eur J Clin Nutr 66, 322-328.

6. Peters J, Sinn N, Campbell K et al. (2012) Parental influences on the diets of 2-5- year-old children: systematic review of interventions. Early Child Dev Care 182, 837-857.

7. Kieft-de Jong JC, de Vries JH, Bleeker SE et al. (2013) Sociodemographic and lifestyle determinants of 'Western-like' and 'Health-conscious' dietary patterns in toddlers. BrJ Nutr 109, 137-147.

8. Mak TN, Prynne CJ, Cole D et al. (2013) Patterns of sociodemographic and food practice characteristics in relation to fruit and vegetable consumption in children: reults from UK National Diet and Nutrition Survey Rolloing Program (2008-2010). Public Health Nutr 16, 1912-1923.

9. Darmon N \& Drewnowski A (2008) Does social class predict diet quality? Am J Clin Nutr 87, 1107-1117.

10. Rosenkranz RR \& Dzewaltowski DA (2008) Model of the home food environment pertaining to childhood obesity. Nutr Rev 66, 123-140.

11. Evans RR, Roy J, Geiger BF et al. (2008) Ecological strategies to promote healthy body image among children. I Sch Health 78, 359-367.

12. Hawkins SS, Cole TJ, Law C et al. (2008) Maternal employment and early childhood overweight: findings from the UK Millennium Cohort Study. Int J Obes (Lond) 32, 30-38.

13. Miller DP \& Han WJ (2008) Maternal nonstandard work schedule and adolescent overweight. Am J Public Health 98, 1495-1502.

14. Morrissey TW, Dunifon RE \& Kalil A (2011) Maternal employment, work schedules, and children's body mass index. Child Dev 82, 66-81.

15. Champion SL, Rumbold AR, Steele EJ et al. (2012) Parental work schedules and child overweight and obesity. Int J Obes (Lond) 36, 573-580.

16. Hawkins SS, Cole TJ, Law C et al. (2009) Examining the relationship between maternal employment and health behaviours in 5-year-old British children. J Epidemiol Community Health 63, 999-1004.

17. Bauer KW, Hearst MO, Escoto K et al. (2012) Parental employment and work-family stress: association with family food environment. Soc Sci Med 75, 496-504.

18. Brown JE, Broom DH, Nicholson JM et al. (2012) Do working mothers raise couch potato kids? Maternal employment and children's lifestyle behaviours and weight in early childhood. Soc Sci Med 70, 1816-1824. 
19. Manios Y, Kourlaba G, Kondaki K et al. (2009) Diet quality of preschoolers in Greece based on the Health Eating Index: the GENESIS Study. J Am Diet Assoc 109, 616-623.

20. Johnson RK, Smiciklas-Wright H, Crouter AC et al. (1992) Maternal employment and the quality of young children's diets: empirical evidence based on the 1987-1988 food consumption survey. Pediatrics 90, 245-249.

21. Leventakoul V, Sarri K, Georgiou V et al. (2016) Early life determinants of dietary patterns in preschool children: Rhea mother-child cohort, Crete, Greece. Eur J Clin Nutr 70, 60-65.

22. McIntosh A, Kubena KS, Tolle G et al. (2011) Determinants of children's use of and time spent in fast-food and full-service restaurants. $J$ Nutr Educ Behav 43, 142-149.

23. Organisation for Economic Co-operation and Development (2015) OECD Family Database: LMF 1.1 - Children in households by employment status. http://www.oecd.org/social/ family/LMF_1_1_Children_in_households_employment_ status.pdf (accessed August 2016).

24. Li J, Johnson SE, Han WJ et al. (2014) Parents' nonstandard work schedules and child well-being: a critical review of the literature. J Prim Prev 35, 53-73.

25. Chiang TL, Lin SJ \& Chang MC (2011) An introduction to the Taiwan Birth Cohort Study. In The Health Profile of Taiwanese Children in the 21st Century, pp. 1-12 [TL Chiang and SJ Lin, editors]. Taipei: Bureau of Health Promotion, Ministry of Health.

26. Mindlin M, Jenkins R \& Law C (2009) Maternal employment and indicators of child health: a systematic review in preschool children in OECD countries. J Epidemiol Community Health 63, 340-350.

27. Ministry of Labor (2016) Statistics of Annual and Weekly Work Hours for Employed Workers in Major Job, and Rates of Part-time Employment. http://www.statdb.mol.gov.tw/ html/nat/104/104006\%E8\%A1\%A81-1.pdf (accessed July 2017).

28. Virudachalam S, Long JA, Harhay MO et al. (2014) Prevalence and patterns of cooking dinner at home in the USA: National Health and Nutrition Examination Survey (NHANES) 2007-2008. Public Health Nutr 17, 1022-1030.

29. Chu YL, Storey KE \& Veugelers PJ (2014) Involvement in meal preparation at home is associated with better diet quality among Canadian children. J Nutr Educ Behav $\mathbf{4 6}$, 304-308.

30. Hu FB (2002) Dietary pattern analysis: a new direction in nutritional epidemiology. Curr Opin Lipidol 13, 3-9.

31. Panagiotakos D (2008) $\alpha$-priori versus $\alpha$-posterior methods in dietary pattern analysis: a review in nutrition epidemiology. Nutr Bull 33, 311-315.

32. Kant AK (2004) Dietary patterns and health outcomes. J Am Diet Assoc 104, 615-635.

33. Hoffman K, Schulze MB, Schienkiewitz A et al. (2004) Application of a new statistical method to derive dietary patterns in nutritional epidemiology. Am J Epidemiol 159, 935-944.

34. Reedy J, Wirfalt E, Flood A et al. (2010) Comparing 3 dietary pattern methods - cluster analysis, factor analysis, and index analysis - with colorectal cancer risk. Am J Epidemiol 171, 479-487.

35. Smith AD, Emmett PM, Newby PK et al. (2013) Dietary patterns obtained through principal components analysis: the effect of input variable quantification. Br J Nutr 109, $1881-1891$.

36. Ministry of Health and Welfare (2012) Daily Dietary Guidelines for Taiwanese. http://www.hpa.gov.tw/Pages/ EBook.aspx?nodeid=1217 (accessed February 2017).

37. Strazdins L, Clements MS, Korda RJ et al. (2006) Unsociable work? nonstandard work schedules, family relationships, and children's well-being. J Marriage Fam 68, 394-410.

38. Cho E \& Ciancetta L (2016) Child outcomes associated with parent-work family experiences. In The Oxford Handbook of Work and Family, pp. 151-164 [TD Allen and LT Eby, editors]. New York: Oxford University Press.

39. Ministry of Education (2017) Regulations of Preschool Education and Care Services. http://law.moj.gov.tw/LawClass/ LawContent.aspx?PCODE $=\mathrm{H} 0070047$ (accessed December 2017).

40. Qian Y \& Sayer LC (2016) Division of labor, gender ideology, and marital satisfaction in East Asia. J Marriage Fam $\mathbf{7 8}, 383-400$.

41. Grosch JW, Caruso CC, Rosa RR et al. (2006) Long hours of work in the US: associations with demographic and organizational characteristics, psychosocial working conditions, and health. Am J Ind Med 49, 943-952.

42. Hill EJ, Hawkins AJ, Ferris AJ et al. (2001) Finding an extra day a week: the positive influence of perceived job flexibility on work and family life balance. Fam Relat 50, 49-58.

43. Baines S \& Gelder U (2003) What is family friendly about the workplace in the home: the case of self-employed parents and their children. New Technol Work Employ 18, 223-234.

44. Jabs J \& Devine CM (2006) Time scarcity and food choices: an overview. Appetite 47, 196-204.

45. Robson SM, Crosby LE \& Stark LJ (2016) Eating dinner away from home: perspectives of middle- to high-income parents. Appetite 96, 147-153.

46. Wu JCL, Bradley RH \& Chiang TL (2012) Cross-border marriage and disparities in early childhood development in a population-based birth cohort study: the mediation of the home environment. Child Care Health Dev 38, 595-603.

47. Bugge $\mathrm{AB} \&$ Almås R (2006) Domestic dinner: representations and practices of a proper meal among young suburban mothers. J Consum Cult 6, 203-228.

48. Marquis M \& Shatenstein B (2005) Food choice motives and the importance of family meals among immigrant mothers. Can J Diet Pract Res 66, 77-82.

49. Hebert JR, Clemow L, Pbert L et al. (1995) Social desirability bias in dietary self-report may compromise the validity of dietary intake measures. Int J Epidemiol 24, 389-398.

50. Li J, Mattes E, Stanley F et al. (2009) Social determinants of child health and well-being. Health Sociol Rev 18, 3-11.

51. Bianchi SM \& Milkie MA (2010) Work and family research in the first decade of the 21st century. J Marriage Fam $\mathbf{7 2}$, $705-725$ 